\title{
lonomidotis mesophila (Ascomycota, Cordieritidaceae), una especie nueva del bosque de niebla en México
}

\section{Ionomidotis mesophila (Ascomycota, Cordieritidaceae), a new species from tropical cloud forest in Mexico}

\section{Acta Botanica Mexicana}

\author{
Marcos Sánchez Flores',2 (D), Michelle Martínez-Pineda² (D), Tania Raymundo2,3 (ID)
}

\begin{abstract}
Resumen:
Antecedentes y Objetivos: Ionomidotis es un género de Ascomicetos que se caracteriza por ascomas solitarios a cespitosos, discoides a espatulados de colores oliváceos a negros, que liberan pigmentos de color púrpura, violeta, oliváceos o verdes en $\mathrm{KOH}$; se encuentra en el orden Helotiales de la clase Leotimycetes. El presente estudio tiene como objetivo describir a lonomidotis mesophila por primera vez para la ciencia.

Métodos: Los especímenes fueron recolectados en 2017 y 2019 en el municipio Honey, Puebla, México. Los ejemplares fueron estudiados de acuerdo con las técnicas tradicionales en micología y depositados en la colección de hongos del herbario de la Escuela Nacional de Ciencias Biológicas del Instituto Politécnico Nacional.

Resultados clave: Se registra por primera vez para México al género lonomidotis y se describe a I. mesophila como especie nueva que se caracteriza por formar apotecios espatulados a cupuliformes, con margen ondulado, de color marrón grisáceo, que en presencia de $\mathrm{KOH}$ al 5\% libera pigmentos de color verde en fresco a marrón oliváceos; paráfisis con ápices redondeados a globosos y ascoporas elipsoides. Crece sobre troncos recién caídos de Quercus en bosque mesófilo de montaña.

Conclusiones: lonomidotis mesophila es una especie nueva para los inventarios del bosque de niebla en México, lo que nos lleva a acelerar esfuerzos por describir la diversidad fúngica del bosque mesófilo de montaña, actualmente definido como un ecosistema en peligro de extinción en el país.
\end{abstract}

Palabras clave: bosque mesófilo, especie nueva, Leotiomycetes, Quercus.

\section{Abstract:}

Background and Aims: The ascomycete genus lonomidotis is characterized by solitary to caespitose ascomata, discoid to spathulate, olive to black, that release purple, violet, olive or green pigments in $\mathrm{KOH}$. It is found in the order Helotiales of the class Leotimycetes. The present study aims to describe lonomidotis mesophila as a new species for science.

Methods: The specimens were collected in 2017 and 2019 in the municipality of Honey, Puebla, Mexico. The samples were studied and determined according to traditional techniques in mycology and were deposited in the fungus collection of the herbarium of the Escuela Nacional de Ciencias Biológicas of the Instituto Politécnico Nacional.

Key results: The genus lonomidotis is registered for the first time from Mexico and $I$. mesophila is decribed as a new species characterized by spathulate to cupuliform apothecia with a wavy, grayish brown margin that, in the presence of $5 \% \mathrm{KOH}$, releases fresh green to olive-brown pigments; paraphyses with rounded to globose apices and ellipsoid ascospores. It grows on recently fallen Quercus in cloud forest.

Conclusions: lonomidotis mesophila is a new species for cloud forest inventories in Mexico. It is necessary to accelerate efforts to inventory and describe the fungal diversity of tropical cloud forest, actually defined as an ecosystem in danger of extintion in the country.

Key words: Leotiomycetes, new species, Quercus, tropical cloud forest.

${ }^{1}$ Instituto Tecnológico de Ciudad Victoria, Tecnológico Nacional de México, Herbario Micológico José Castillo, Boulevard Emilio Portes Gil No. 1301, 87010 Ciudad Victoria, Tamaulipas, México.

${ }^{2}$ Instituto Politécnico Nacional, Escuela Nacional de Ciencias Bilógicas, Laboratorio de Micología, Prolongación de Carpio y Plan de Ayala, Santo Tomás, Alcaldía Miguel Hidalgo, 11340 Cd. Mx., México.

${ }^{3}$ Autor para la correspondencia: traymundoo@ipn.mx; raymundot.tr@gmail.com
Recibido: 4 de noviembre de 2020.

Revisado: 12 de diciembre de 2020

Aceptado por Marie-Stéphanie Samain: 19 de marzo de 2021.

Publicado Primero en línea: 8 de abril de 2021.

Publicado: Acta Botanica Mexicana 128 (2021).
Citar como: Sánchez Flores, M., M. Martínez-Pineda y T. Raymundo. 2021. Ionomidotis mesophila (Ascomycota, Cordieritidaceae), una especie nueva del bosque de niebla en México. Acta Botanica Mexicana 128: e1812. DOI: https://doi.org/10.21829/abm128.2021.1812 


\section{Introducción}

El género lonomidotis E.J. Durand ex Thaxt. pertenece a la familia Cordieritidaceae, orden Helotiales, clase Leotiomycetes, Phylum Ascomycota. Durand (1923) caracterizó al género por formar ascomas superficiales, solitarios o cespitosos, irregulares y alargados cuando maduran, colores violeta, oliva a negros, consistencia frágil, que libera pigmentos generalmente color púrpura, violeta oscuro, verde inglés o verde oliva con $\mathrm{KOH}$; excípulo medular constituido por hifas entrelazadas; excípulo ectal constituido por células pseudoparenquimatosas; ascas claviforme-cilíndricas con poro inamiloide en Melzer; esporas pequeñas, hialinas, elipsoides a oblongas. El hábito de las especies de lonomidotis es lignícola-saprobia y se han reportado sobre Carpinus betulus L., Fagus sylvatica L., Quercus alba L., Quercus sp., Ulmus sp. y en madera en descomposición en bosques templados. Actualmente se conocen solo cuatro taxones válidos (Index Fungorum, 2020;
Wijayawardene et al., 2020): lonomidotis fulvotingens (Berk. \& M.A. Curtis) E.K. Cash descrita por Berkeley y Curtis (1875), I. irregularis (Schwein.) E.J. Durand por Schweinitz (1832), ambas registradas del estado de Pennsylvania, EUA, I. portoricensis Seaver descrita de Puerto Rico por Seaver (1925) e I. sprucei (Berk.) E. J. Durand descrita de la Amazonia, Brasil por Berkeley (1856). El género aún no se ha reportado para el país. El presente estudio tiene como objetivo describir una especie nueva del género lonomidotis para la ciencia.

\section{Materiales y Métodos}

Se recolectaron especímenes en el municipio Honey, localizado en la Sierra Norte de Puebla dentro de la Sierra Madre Oriental (Fig. 1). En esta región se conserva $47-50 \%$ de vegetación natural que, en su mayoría, corresponde a bosque mesófilo de montaña en un rango altitudinal de 800 hasta 1350 m s.n.m. (Reyes-Díaz, 2003). Los ejemplares se deposi-

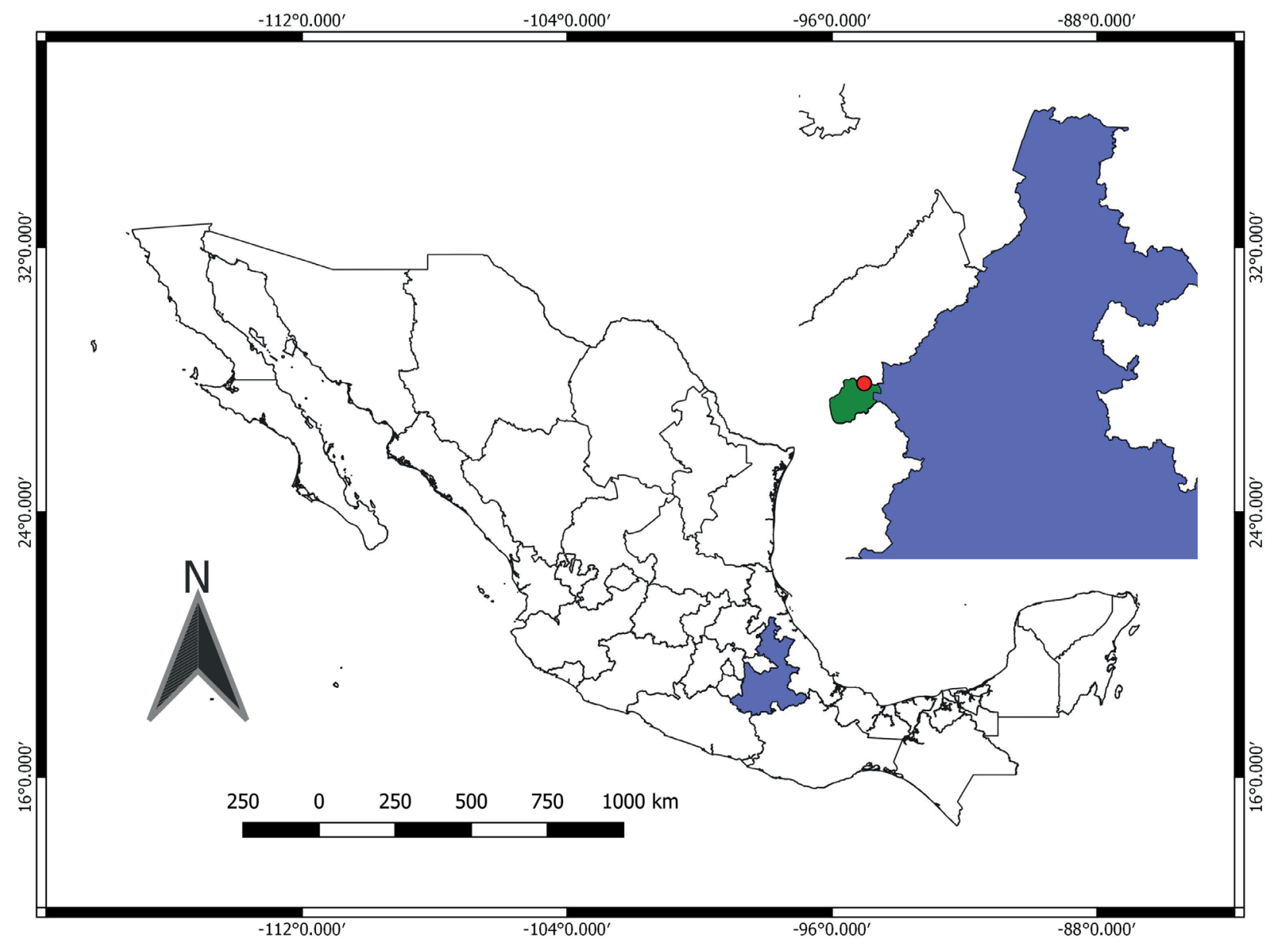

Figura 1: Localidad tipo (punto rojo) de lonomidotis mesophila Sánchez-Flores, Martínez-Pineda \& Raymundo en Honey, Puebla, México. 
taron en la colección de hongos del herbario de la Escuela Nacional de Ciencias Biológicas del Instituto Politécnico Nacional (ENCB).

El material colectado se examinó siguiendo las técnicas tradicionales de la micología propuestas por Cifuentes et al. (1986). Los especímenes se caracterizaron en fresco, describiendo los caracteres macroscópicos de acuerdo con su tamaño, forma, color y, en algunos casos, textura. La terminología hace referencia al diccionario ilustrado de micología (Ulloa y Hanlin, 2006), mientras que los colores se indican según la tabla de color Kornerup y Wanscher (1978). Se realizaron cortes longitudinales de los apotecios, se rehidrataron con alcohol al $70 \%$, se agregó $\mathrm{KOH}$ al $5 \%$ y $\mathrm{NaOH}$ al $10 \%$. Algunas muestras se tiñeron con reactivo Melzer para observar amiloidia de las ascas. Se analizaron y caracterizaron las estructuras microscópicas de los diferentes excípulos, paráfisis, ascas y ascosporas, utilizando un microscopio óptico (MO) (K-7 Zeiss, Jena, Alemania), los cuales se ilustraron en las descripciones y fotografiaron con una cámara D-7000 y un lente DX Micor 85 mm (Nikon, Tokio, Japón). Para examinar el detalle de ornamentación se utilizó microscopía electrónica de barrido (MEB) (JSM-5800LV, Jeol, Peabody, Massachusetts, EUA). Para determinar el género se utilizó la obra de Durand (1923).

\section{Resultados}

Se registra el género lonomidotis por primera vez para México y se propone lonomidotis mesophila Sánchez-Flores, Martínez-Pineda \& Raymundo como nueva especie para la ciencia. A continuación, se presenta una clave para identificar las especies de lonomidotis.

\section{Clave para las especies de lonomidotis}

1a. Ascomas liberan pigmentos en $\mathrm{KOH}$ al $5 \%$ 2

1b. Ascomas no liberan pigmentos en $\mathrm{KOH}$ al $5 \%$

I. portoricensis Seaver

2a. Reacción en $\mathrm{KOH}$ colores violetas a púrpuras ............... 3

2b. Reacción en $\mathrm{KOH}$ otros colores (amarillento, verdes, oliváceos, marrones a rojizos)

3a. Pigmentos color violeta, paráfisis con ápices lanceolados; ascosporas 8-10 $\times 3-4 \mu \mathrm{m}$, oblongas

I. irregularis (Schwein.) E.J. Durand

3b. Pigmentos color púrpura, paráfisis con ápices nodulo- sos; ascosporas 5-6 $6 \times 3 \mu \mathrm{m}$, elipsoides I. sprucei (Berk.) E.J. Durand

4a. Pigmentos color verde inglés a verde oliva, ascomas espatulados, cupuliformes, estípitados; ascosporas (3.2)4.85.6×1-1.5 $\mu \mathrm{m}$, elipsoides I. mesophila Sánchez-Flores, Martínez-Pineda \& Raymundo 4b. Pigmentos color amarillento, leonado brillante a marrón rojizo, ascomas discoides a cupuliformes, sésiles; ascosporas $5-8 \times 1-2 \mu \mathrm{m}$, oblongas

I. fulvotingens (Berk. \& M.A. Curtis) E.K. Cash

\section{Taxonomía}

Ascomycota

\section{Leotiomycetes}

Helotiales

\section{Cordieritidaceae}

Ionomidotis mesophila Sánchez-Flores, Martínez-Pineda \& Raymundo, sp. nov. Figs. 2-4.

TIPO: MÉXICO. Puebla, municipio Honey, San Pedro Chila, 1309 m s.n.m., 20¹7'38.78"N, 98¹324.69"O, 17.IX.2017, M. Sánchez 940 (holotipo: ENCB!), Mycobank: MB 838437.

Apotecio $20-50 \mathrm{~mm}$ longa, $10-30 \mathrm{~mm}$ latum, ascis (57)60-73 × 3.2-4 $\mu \mathrm{m}$, cylindratis, octosporicas, uniseriadas; ascosporae (3.2)4.8-5.6 $\times 1$ 1-1.5 $\mu \mathrm{m}$, elipsoides, hyalinis, duo gutulas. De lignis apparet cecidere recentes Quercus.

Ascomas cespitosos a gregarios, apotecios 20-50 mm de largo, 10-30 mm de ancho, espatulados, negro, textura rugosa, consistencia carnosa a dura, margen del apotecio irregular, cupuliforme, alargándose hasta formar un estípite, himenio color marrón-grisáceo (27F1), libera pigmentos verde inglés (27B6) a verde oliváceo (27D6) en fresco, oliváceo a marrón en especímenes deshidratados en $\mathrm{KOH}$ al $5 \%$, color marrón en $\mathrm{NaOH}$ al $10 \%$, vináceos en alcohol al 70\%; excípulo ectal 24-40 $\mu \mathrm{m}$ de grosor, textura globular forma- 

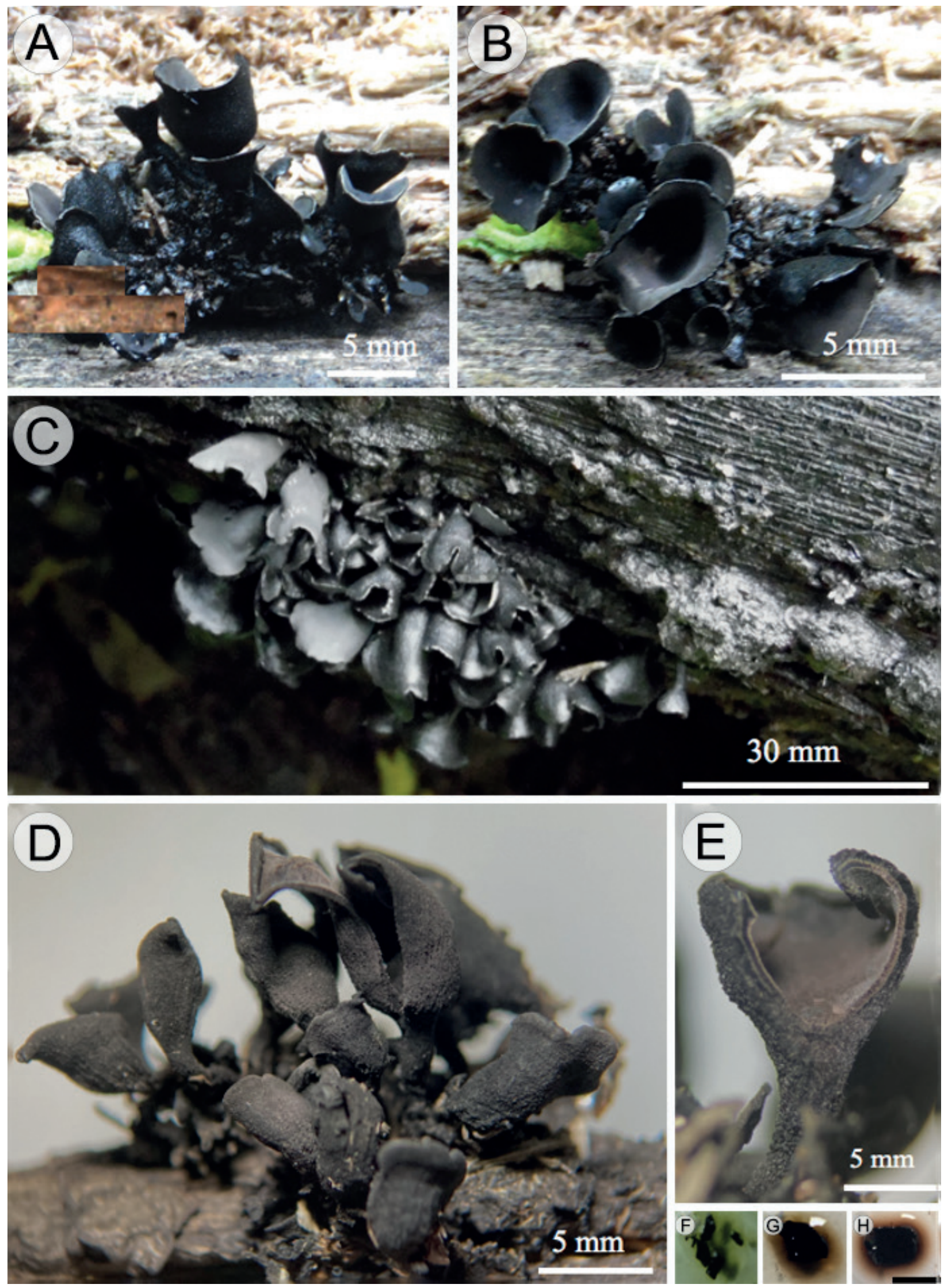

Figura 2: Ionomidotis mesophila Sánchez-Flores, Martínez-Pineda \& Raymundo: A, B. ascomas en fresco; C. ascomas sobre tronco de Quercus L.; D. ascomas deshidratados; E. corte longitudinal del apotecio deshidratado; F. reacción del himenio en $\mathrm{KOH}$ al 5\%; G. reacción del himenio en NaOH al $10 \%$; H. reacción del himenio en alcohol al $70 \%$. 

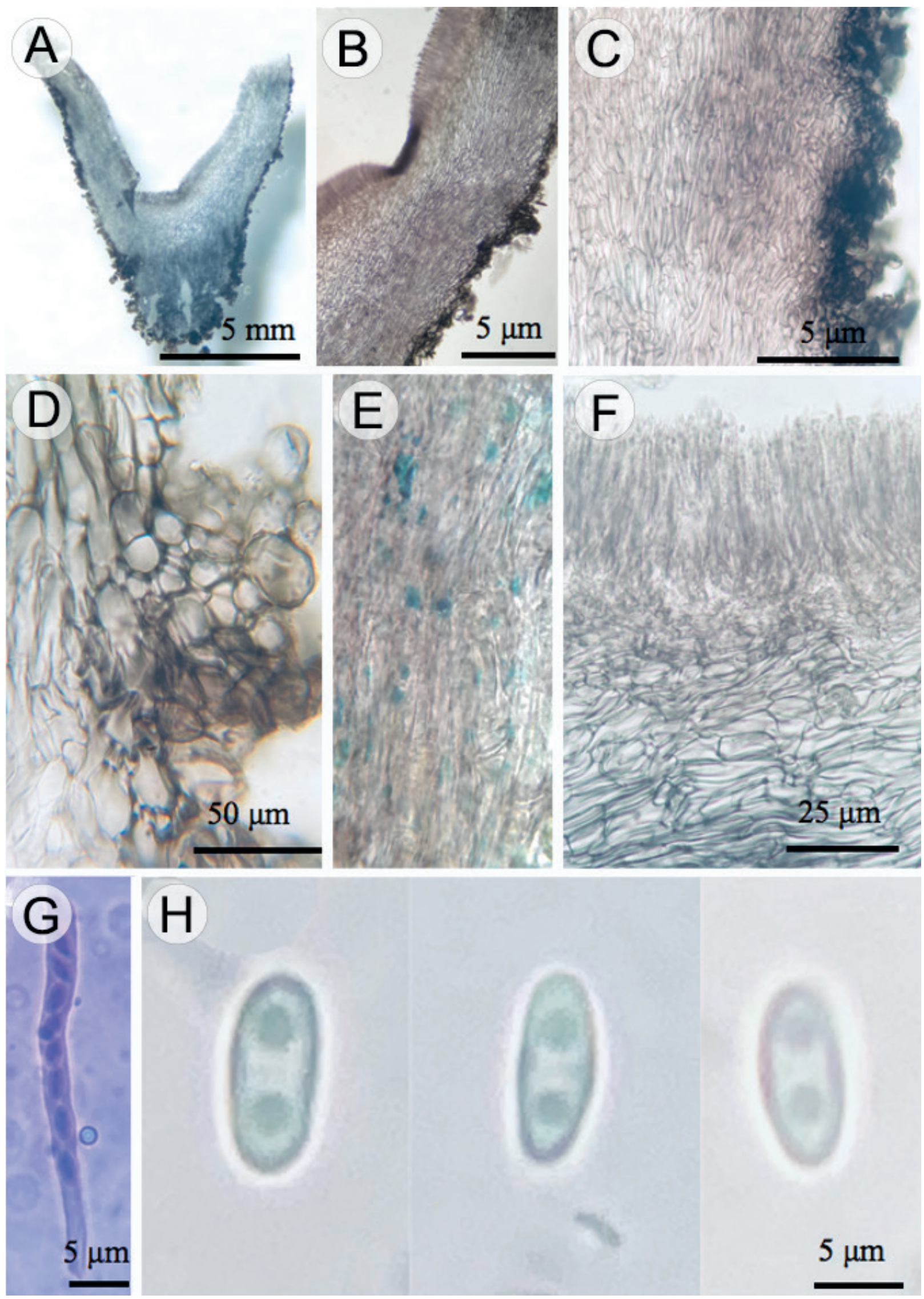

Figura 3: Ionomidotis mesophila Sánchez-Flores, Martínez-Pineda \& Raymundo: A. corte longitudinal del ascoma; B. corte del apotecio; C. excípulo ectal y medular del estípite; D. excípulo ectal; E. pigmentos verdes en excípulo medular; F. himenio; G. asca; H. ascosporas. 


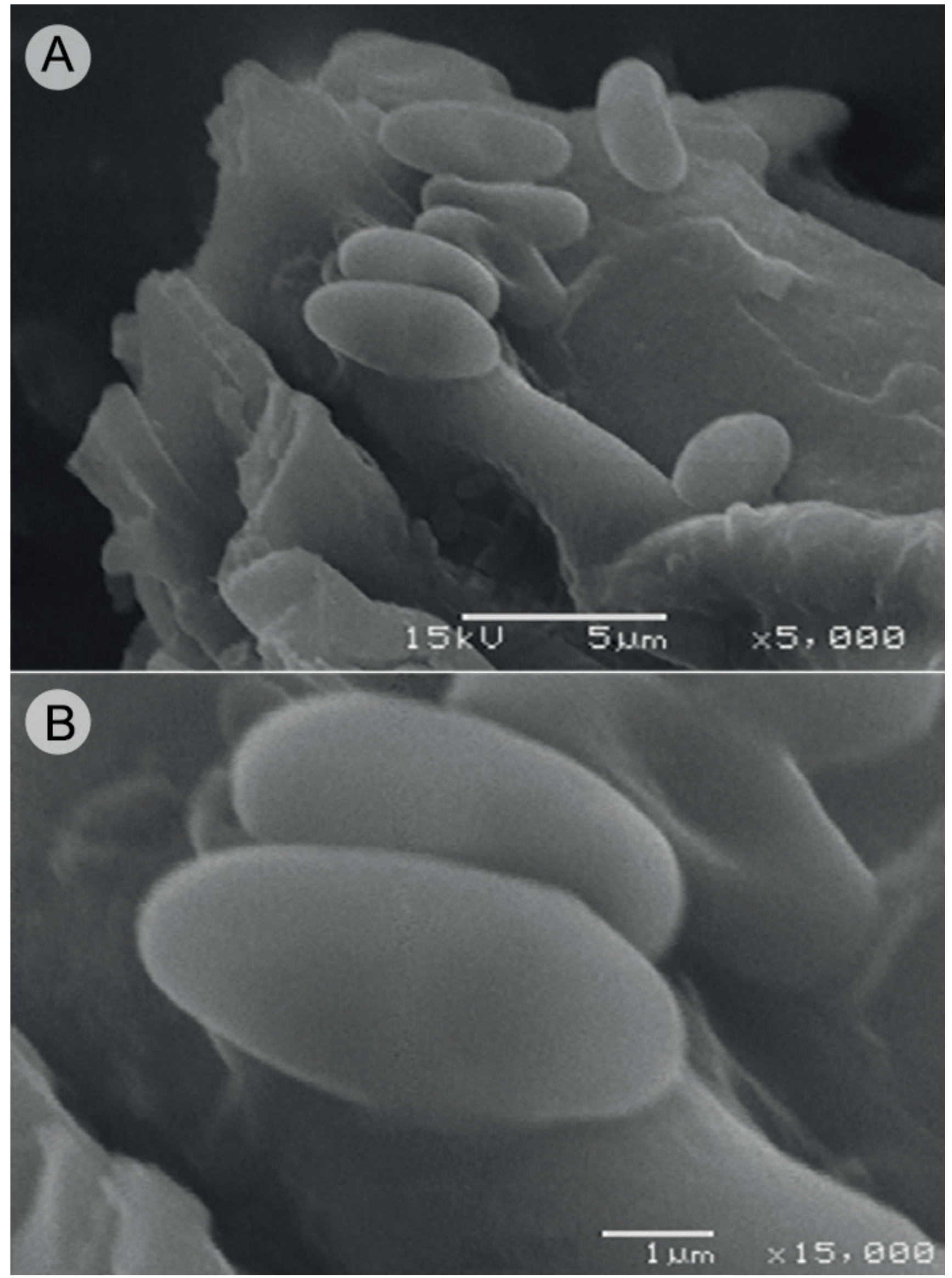

Figura 4: Ionomidotis mesophila Sánchez-Flores, Martínez-Pineda \& Raymundo: A, B. ascosporas en MEB. 
do por células 10-25 × 8-18 $\mu \mathrm{m}$, marrón (30F4), pared delgada a gruesa; excípulo medular 114-140 $\mu \mathrm{m}$ de grosor, textura intrincada, formada por hifas paralelas a ligeramente entrelazadas, 5.6-8 $\mu \mathrm{m}$ de diámetro, hifas color lila (17D5) a azul náutico (20F6), presentando pigmentos color verde inglés (25C7); subhimenio 106.4-114 $\mu \mathrm{m}$ de grosor; paráfisis $60-80 \times 1.6-2.4 \mu \mathrm{m}$ de diámetro, filiformes, hialinas, con el ápice redondeado a capitado, septada hacia la base; ascas (57-)60-73 × 3.2-4 $\mu \mathrm{m}$, cilíndricas, octosporadas, uniseriadas, poro apical inamiloide; ascosporas (3.2-)4.8-5.6 $\times$ 1.0-1.5 $\mu \mathrm{m}$, elipsoides, hialinas, pared delgada y lisa, con dos gútulas, lisas vistas en MEB.

Hábito: crece sobre corteza de Quercus L. recién caído en bosque mesófilo de montaña.

Distribución: conocido únicamente del municipio de la localidad tipo. El género se registra por primera vez para México

Etimología: hace referencia al tipo de vegetación en el que se encontró.

Material estudiado: MÉXICO. Puebla, municipio Honey, Cascadas Arcoíris, Rincón de Chila, $2144 \mathrm{~m}$ s.n.m., 20¹5'32.4"N, 98¹4'48.3"O, 24.VII.2019, M. Martínez-Pineda 23 (ENCB); San Pedro Chila, 1309 m s.n.m., 20¹7'38.78' N, 98¹3'24.69"O, 17.IX.2017, M. Sánchez 941 (ENCB).

Notas taxonómicas: lonomidotis mesophila es una especie que se caracteriza por apotecios espatulados con margen ondulado, libera pigmentos en $\mathrm{KOH}$ al 5\%, color verde inglés en fresco a oliváceo en seco, ascosporas elipsoides de 4.8-5.6 × 1.0-1.5 $\mu \mathrm{m}$, hialinas, bigutúladas. Una especie afín es $I$. irregularis, la cual se diferencia por presentar ascomas alargados e irregularmente lobulados o lacerados en el margen color marrón obscuro con himenio negro, liberando pigmentos violetas en $\mathrm{KOH}$ al 5\%, paráfisis con ápice lanceolado, ascosporas de 8-10 × 3-4 $\mu \mathrm{m}$ (Durand, 1923). Běták et al. (2012) la registraron en la Republica Checa con ascosporas de 7-9 × 2.8-3.5 $\mu \mathrm{m}$. Ionomidotis sprucei se diferencia por apotecios en forma de copa, estipitados, color vináceo obscuro, furfuráceos, que desprenden pigmentos color púrpura en $\mathrm{KOH}$ al $5 \%$, paráfisis nodulosas y ascosporas más anchas de 5-6 × $3 \mu \mathrm{m}$ (Durand, 1923). lonomidotis portoricensis se diferencia por un ascoma sésil o subsésil de contorno irregular color marrón claro, furfuráceo que no libera pigmentos en $\mathrm{KOH}$ al $5 \%$, paráfisis con ápices nodulosos, ascosporas de 4-6 × $2 \mu \mathrm{m}$, elipsoides (Seaver, 1925). Ionomidotis fulvotingens presenta apotecios cupuliformes a discoides, recurvados hacia el margen, oliváceos, pardo-verdoso, marrón verdoso, marrón rojizo (Cash, 1939), que desprende pigmentos color leonado brillante, pulverulentos, ascosporas 5-8 × 1-2 $\mu \mathrm{m}$ (Berkeley y Curtis, 1875). Este género es de hábito saprobio, presenta cinco especies las cuales tienen distribución restringida en el continente americano, excepto Ionomidotis irregularis que ha sido citada de Austria (Zhuang, 1988), Eslovaquia, Polonia y la República Checa (Běták et al., 2012; Cuadro 1). Posiblemente el género presente una mayor riqueza de especies; no obstante, es difícil cuantificar la diversidad en estos taxa ante la pérdida de los ecosistemas templados que en el presente son de los más vulnerables por el cambio climático. Tal es el caso del bosque mesófilo en donde se desarrolla lonomidotis mesophila, la cual se adjunta a los inventarios de ascomicetos de este tipo de vegetación; con la que actualmente se tendrían un total de 157 especies considerando las aportaciones de Raymundo et al. (2020) y Sánchez-Flores et al. (2020).

\section{Contribución de autores}

MSF y TR concibieron y diseñaron el estudio. MSF y MMP realizaron la recolección de la especie. MSF, MMP y TR contribuyeron a la adquisición de datos importantes para el trabajo. Las fotografías fueron tomadas por TR y MSF. MSF y TR escribieron el manuscrito con ayuda de MMP. Todos los autores contribuyeron a la discusión, revisión y aprobación del manuscrito final.

\section{Financiamiento}

Este estudio fue apoyado por el Instituto Politécnico Nacional (IPN), a través de la Secretaria de Investigación y Posgrado (SIP) en los proyectos 20200248, 20210315. TR recibió becas de la Secretaria de Investigación y Posgrado y del Sistema Nacional de Investigadores. 
Cuadro 1: Comparación de especies del género lonomidotis E.J. Durand ex Thaxt. hasta ahora registradas a escala mundial, basado en las obras de Berkeley y Curtis (1875), Cash (1939), Durand (1923) y Seaver (1925).

\begin{tabular}{|c|c|c|c|c|c|}
\hline & $\begin{array}{l}\text { I. mesophila } \\
\text { Sánchez-Flores, } \\
\text { Martínez-Pineda \& } \\
\text { Raymundo }\end{array}$ & $\begin{array}{l}\text { I. fulvotingens (Berk. \& } \\
\text { M.A. Curtis) E.K. Cash }\end{array}$ & $\begin{array}{l}\text { I. irregularis (Schwein.) } \\
\text { E.J. Durand }\end{array}$ & I. portoricensis Seaver & $\begin{array}{l}\text { I. sprucei (Berk.) E.J. } \\
\text { Durand }\end{array}$ \\
\hline Apotecio & $20-50 \times 10-30 \mathrm{~mm}$ & $1-10 \mathrm{~mm}$ & $(10-) 15-30 \mathrm{~mm}$ & $5-8 \mathrm{~mm}$ & 3-5 mm \\
\hline $\mathrm{KOH}$ al $5 \%$ & $\begin{array}{l}\text { + verde inglés a verde } \\
\text { oliva }\end{array}$ & $\begin{array}{l}\text { + amarillento, leonado } \\
\text { brillante a marrón } \\
\text { rojizo }\end{array}$ & + violeta & no libera pigmentos & + púrpura \\
\hline Paráfisis & $\begin{array}{l}\text { 1.6-2.4 } \mu \mathrm{m} \text {, ápice } \\
\text { redondeado }\end{array}$ & $\begin{array}{l}\text { no se indica en la } \\
\text { descripción }\end{array}$ & $\begin{array}{l}\text { 3-4 } \mu \mathrm{m} \text {, ápice } \\
\text { lanceolado }\end{array}$ & ápice noduloso & ápice noduloso \\
\hline Asca & $(57-) 60-73 \times 3.2-4 \mu \mathrm{m}$ & $40-50 \times 3.5-5 \mu \mathrm{m}$ & $50-70 \times 4-5 \mu \mathrm{m}$ & $30 \times 5 \mu \mathrm{m}$ & $65 \mu \mathrm{m}$ \\
\hline Ascosporas & $\begin{array}{l}(3.2-) 4.8-5.6 \times 1-1.5 \\
\mu m, \text { elipsoides }\end{array}$ & $\begin{array}{l}5-8 \times 1-2 \mu \mathrm{m}, \\
\text { oblongas }\end{array}$ & $\begin{array}{l}\text { 8-10 } \times 3-4 \mu \mathrm{m} \text {, } \\
\text { oblongas }\end{array}$ & $\begin{array}{l}4-6 \times 2 \mu \mathrm{m}, \\
\text { elipsoides }\end{array}$ & $\begin{array}{l}5-6 \times 3 \mu \mathrm{m} \\
\text { elipsoides }\end{array}$ \\
\hline Hospedero & sobre Quercus L. & sobre madera seca & Fagus sylvatica L. & sobre madera & sobre madera \\
\hline Distribución & Puebla, México & $\begin{array}{c}\text { Pennsylvania, Estados } \\
\text { Unidos de América }\end{array}$ & $\begin{array}{l}\text { Pennsylvania, Estados } \\
\text { Unidos de América }\end{array}$ & Puerto Rico & Amazonia, Brasil \\
\hline
\end{tabular}

\section{Agradecimientos}

MSF, MMP y TR agradecen a las autoridades de Las Cascadas Arcoíris por el apoyo brindado para la realización de este estudio.

\section{Literatura citada}

Berkeley, M. J. 1856. Decades of Fungi. Decades LVII-LVIII. Rio Negro fungi. Hooker's Journal of Botany and Kew Garden Miscellany 8: 193-200.

Berkeley, M. J. y M. A. Curtis. 1875. Notices of North American Fungi. Grevillea 4(29): 1-16.

Běták, J., K. Pärtel y M. Kř́iž. 2012. Ionomidotis irregularis (Ascomycota, Helotiales) in the Czech Republic with comments on its distribution and ecology in Europe. Czech Mycology 64(1): 79-92. DOI: https://doi.org/10.33585/cmy.64109

Cash, E. K. 1939. Botany. Some Georgia Discomycetes. Journal of the Washington Academy of Sciences 29(2): 47-51.

Cifuentes, J., M. Villegas, L. Pérez-Ramírez. 1986. Hongos. In: Lot, A. y F. Chiang (eds.). Manual de Herbario. Consejo Nacional de la Flora en México, A.C. México, D.F., México. Pp. 55-64.

Durand, E. J. 1923. The genera Midotis, Ionomidotis and Cordierites. Proceedings of the American Academy of Arts and Sciences 59(1): 3-18. DOI: https://doi.org/10.2307/20026049
Index Fungorum. 2020. Index Fungorum base de datos. http:// www.indexfungorum.org/Names/Names.asp (consultado octubre de 2020).

Kornerup, A. y J. H. Wanscher. 1978. Methuen Handbook of Colour. 3a ed. Eyre Methuen. London, UK. 252 pp.

Raymundo, T., R. Valenzuela, J. C. Ramírez-Martínez, M. MartínezPineda, A. Cobos Villagrán, A. Trejo Arana, M. Sánchez-Flores, A. D. Gay-González e I. Luna Vega. 2020. New records of Ascomycetes from the Tropical Mountane Cloud Forests of eastern Mexico. Phytotaxa 454(3):161-185. DOI: https://doi. org/10.11646/phytotaxa.454.3.1

Reyes-Díaz, M. 2003. Estudio de la vegetación de los municipios de Honey y Pahuatlán de la Sierra Norte de Puebla, Puebla. Tesis de licenciatura. Facultad de Ciencias, Universidad Nacional Autónoma de México. Cd. Mx., México. 81 pp.

Sánchez Flores, M., R. Valenzuela, M. A. Hernández-Muñoz, J. García Jiménez, M. Martínez-Pineda y T. Raymundo. 2020. Ascomicetos del bosque mesófilo de montaña de Honey, Puebla de los Ángeles, México. Acta Botanica Mexicana 127: e1719. DOI: https://doi.org/10.21829/abm127.2020.1719

Seaver, F. J. 1925. Studies in tropical ascomycetes-III. Porto Rican cup-fungi. Mycologia 17(2): 45-50. DOI: https://doi.org/10.1 080/00275514.1925.12020455 
Schweitnitz, L. D. 1832. Sypnopsis fungorum in America Boreali media degentium. Transactions American Philosophical Socitey 4(2): 141-316. DOI: https://doi.org/10.2307/1004834

Ulloa, M. y R. T. Hanlin. 2006. Nuevo diccionario ilustrado de Micología. APS Press. St. Paul, USA. 615 pp.

Wijayawardene, N. N., K. D. Hyde, L. K. T. Al-Ani, L. Tedersoo, D. Haelewaters, K. C. Rajeshkumar, R. L. Zhao, A. Aptroot, D. V. Leontyev, R. K. Saxena, Y. S. Tokarev, D. Q. Dai, P. M. Letcher, S. L. Stephenson, D. Ertz, H. T. Lumbsch, M. Kukwa, I. V. Issi, H. Madrid, A. J. L. Phillips, L. Selbmann, W. P. Pfliegler, E. Horváth, K. Bensch, P. M. Kirk, K. Kolaříková, H. A. Raja, R. Radek, V. Papp, V. Dima, J. Ma, E. Malosso, S. Takamatsu, G. Rambold, P. B. Gannibal, D. Triebel, A. K. Gautam, S. Avasthi, S. Suetrong, E. Timdal, S. C. Fryar, G. Delgado, M. Réblová, M. Doilom, S. Dolatabadi, J. Pawłowska, R. A. Humber, R. Kodsueb, I. Sánchez-Castro, B. T. Goto, D. K. A. Silva, F. A. de Souza, F. Oehl, G. A. da Silva, I. R. Silva, J. Błaszkowski, K. Jobim, L. C. Maia, F. R. Barbosa, P. O. Fiuza, P. K. Divakar, B. D. Shenoy, R. F. Castañeda-Ruiz, S. Somrithipol, A. A. Lateef, S. C. Karunarathna, S. Tibpromma, P. E. Mortimer, D. N. Wanasinghe, R. Phookamsak, J. Xu, Y. Wang, F. Tian, P. Alvarado, D. W. Li, I. Kušan, N. Matočec, S. S. N. Maharac- hchikumbura, M. Papizadeh, G. Heredia, F. Wartchow, M. Bakhshi, E. Boehm, N. Youssef, V. P. Hustad, J. D. Lawrey, A. L. C. M. A. Santiago, J. D. P. Bezerra, C. M. Souza-Motta, A. L. Firmino, Q. Tian, J. Houbraken, S. Hongsanan, K. Tanaka, A. J. Dissanayake, J. S. Monteiro, H. P. Grossart, A. Suija, G. Weerakoon, J. Etayo, A. Tsurykau, V. Vázquez, P. Mungai, U. Damm, Q. R. Li, H. Zhang, S. Boonmee, Y. Z. Lu, A. G. Becerra, B. Kendrick, F. Q. Brearley, J. Motiejūnaitè, B. Sharma, R. Khare, S. Gaikwad, D. S. A. Wijesundara, L. Z. Tang, M. Q. He, A. Flakus, P. Rodriguez-Flakus, M. P. Zhurbenko, E. H. C. McKenzie, M. Stadler, D. J. Bhat, J. K. Liu, M. Raza, R. Jeewon, E. S. Nassonova, M. Prieto, R. G. U. Jayalal, M. Erdoğdu, A. Yurkov, M. Schnittler, O. N. Shchepin, Y. K. Novozhilov, A. G. S. Silva-Filho, P. Liu, J. C. Cavender, Y. Kang, S. Mohammad, L. F. Zhang, R. F. Xu, Y. M. Li, M. C. Dayarathne, A. H. Ekanayaka, T. C. Wen, C. Y. Deng, O. L. Pereira, S. Navathe, D. L. Hawksworth, X. L. Fan, L. S. Dissanayake, E. Kuhnert, H. P. Grossart y M. Thines. 2020. Outline of Fungi and fungus-like taxa. Mycosphere 11(1): 1060-1456. DOI: https://doi.org/10.5943/mycosphere/11/1/8

Zhuang, W. Y. 1988. Studies on some discomycete genera with an ionomidotic reaction: Ionomidotis, Poloniodiscus, Cordierites, Phyllomyces and Ameghiniella. Mycotaxon 31: 261-298. 